\title{
MAXIMUM LIKELIHOOD ESTIMATION IN LOCATION-SCALE FAMILIES USING VARIED L RANKED SET SAMPLING
}

\author{
Amer I. Al-OMARI*
}

\begin{abstract}
Recently, a generalized ranked set sampling (RSS) scheme has been introduced which encompasses several existing RSS schemes, namely varied L RSS (VLRSS), and it provides more precise estimators of the population mean than the estimators with the traditional simple random sampling (SRS) and RSS schemes. In this paper, we extend the work and consider the maximum likelihood estimators (MLEs) of the location and scale parameters when sampling from a location-scale family of distributions. In order to give more insight into the performance of VLRSS with respect to SRS and RSS schemes, the asymptotic relative precisions of the MLEs using VLRSS relative to that using SRS and RSS are compared for some usual location-scale distributions. It turns out that the MLEs with VLRSS are more precise than those with the existing sampling schemes.
\end{abstract}

Mathematics Subject Classification. 62D05.

Received August 2, 2020. Accepted October 28, 2020.

\section{INTRODUCTION}

In many agricultural, biological, environmental and ecological studies, the main focus is on the development of cost-effective, well-designed and efficient sampling schemes that could help us in achieving observational economy. The traditional ranked set sampling (RSS) method is one of those cost-effective sampling methods that can help us in accomplishing such objectives at an affordable cost. In most of the environmental, biomedical and ecological studies, there are situations where taking the actual measurement is costly, destructive and timeconsuming whereas ranking a small set of selected units is relatively easy and reliable. For example, hazardous waste sites with different contamination levels can be ranked by a visual inspection of soil discoloration but actual measurements of toxic chemical is expensive and time-consuming.

The RSS scheme was first introduced by McIntyre [19] for estimating mean pasture and forage yields. The mathematical background of the RSS scheme was developed by Takahasi and Wakimoto [25]. They proved that the sample mean with RSS is an unbiased estimator of the population mean, and it is more efficient than the sample mean based on simple random sampling (SRS). In a later study, Dell and Clutter [11] further proved that, despite the presence of ranking errors, the RSS mean still remains an unbiased estimator of the population mean, and it is at least as efficient as the mean estimator with SRS. In the last decades, there have been new advancements and improvements in the traditional RSS scheme. Samawi et al. [22] and Muttlak [20] suggested

Keywords. Asymptotic relative precision, fisher information, location-scale family, maximum likelihood estimator, simple random sampling, varied $\mathrm{L}$ ranked set sampling.

Department of Mathematics, Faculty of Science, Al Al-Bayt University, Mafraq 25113, Jordan.

* Corresponding author: alomari_amer@yahoo.com 
extreme RSS (ERSS) and median RSS (MRSS) schemes for estimating the population mean, respectively. A generalized RSS scheme was suggested by Al-Naseer [1] that is based on the idea of L moments, namely L RSS (LRSS), for estimating the population mean. The LRSS scheme encompasses several existing RSS schemes, including RSS, MRSS, quartile RSS schemes. Jemain et al. $[17,18]$ suggested multistage extreme and median ranked set samples for estimating the population mean, respectively. Al-Omari and Raqab [6] proposed truncation-based RSS (TBRSS) for estimating the population mean and median. Haq et al. [13] proposed partial RSS (PRSS) scheme for estimating the population mean, median and variance. The PRSS scheme is a costefficient alternative to the RSS scheme. Recently, Haq et al. [15] suggested another variation of the RSS scheme, namely varied LRSS (VLRSS), that encompasses several existing RSS schemes. The VLRSS scheme is not only a cost-efficient alternative to the traditional RSS scheme but it also provides a more precise estimator of the population mean than those with the existing RSS schemes. Haq et al. [15] considered the VLRSS Al-Omari and Al-Nasser [5] suggested ratio estimation using multistage median ranked set sampling. Al-Omari and Al-Nasser [7] considered the problem of goodness of fit tests for logistic distribution based on Phi-divergence. Zamanzade and Al-Omari [26] suggested a new modification of ranked set sampling for estimating the population mean and variance. Recently, Al-Nasser and Al-Omari [2] introduced the minimax ranked set sampling method for estimating the population mean. Haq and Al-Omari [12] proposed a new Shewhart control chart for monitoring process mean using partially ordered judgment subset sampling. Al-Omari [4] investigated the mean estimation using modified robust extreme ranked set sampling method. Zamanzade and Mahdizadeh [27] used the ranked set sampling method with extreme ranks in estimating the population proportion. Al-Omari et al. [8] studied the estimation of the stress-strength reliability for exponentiated Pareto distribution using median and ranked set sampling methods. Rui et al. [21] considered ranked set sampling design for estimating the parameter of the log-extended exponential-geometric distribution. Samuh et al. [23] investigated the estimation of the parameters of the new Weibull-Pareto distribution using ranked set sampling. Haq et al. [16] suggested a new modification of the RSS called as paired double ranked set sampling. Al-Nasser et al. [3] suggested extreme ranked repetitive sampling control charts and Haq et al. [14] proposed mixed ranked set sampling method.

The VLRSS scheme is as follows. Select the value of the VLRSS coefficient $k=[m \alpha]$ such that $0 \leq \alpha<0.5$, where $[t]$ is a largest integer value less than or equal to $t$, and $m$ is the set size. Identify $2 k m_{1}$ units from the target population, and partition these units into $2 k$ sets, each of size $m_{1}$ units. Rank the units within each set with respect to the study variable or by an inexpensive method. It is also possible to rank the values of the study variable by using information on the ranks of the auxiliary variable provided there exists sufficient correlation between the study and auxiliary variables. Select the $v$ th and $\left(m_{1}-v+1\right)$ th smallest ranked units from the first and last $k$ sets, respectively. In order to select the remaining $(m-2 k)$ units, identify $m(m-2 k)$ units from the target population. Randomly partition these units into $m-2 k$ sets, each of size $m$ units. Then select the $i$ th smallest ranked unit from the $(i+k)$ th set for $i=k+1, \ldots, m-k$. This completes one cycle of a varied $\mathrm{L}$ ranked set sample of size $m$. The whole procedure can be repeated $r$ times to get a varied ranked set sample of size $m$ with total sample size $n=m r$ units. Note here that $v$ is a positive integer, i.e., $v=1,2, \ldots,\left[m_{1} / 2\right]$, and $m_{1} \geq m$ or $m_{1}<m$ depending on the time, cost- or budget-constraints. It is interesting to note that the traditional RSS, ERSS, MRSS, LRSS, TBRSS, and PRSS schemes are special cases of the VLRSS schemes. For more details we refer to Haq et al. [15].

In recent years, there have been a considerable research on the maximum likelihood estimators (MLEs) of the location and scale parameters under the RSS schemes. Stokes [24] examined both maximum likelihood and best linear unbiased estimator of the location and scale parameters for a specific family of distributions using RSS. A modified MLE (MMLE) using RSS for estimating general parameters of a location-scale distribution was suggested by Zheng and Al-Saleh [28]. Balci et al. [9] derived the MMLEs for the population mean and variance under RSS and showed their superiority over the usual RSS estimators. Chen et al. [10] considered MLE of the scale parameter of a scale family of distributions using moving extremes RSS (MERSS) scheme. They showed that the MLE based on MERSS is considerably better than that based on SRS. 
In this paper, we extend the work on VLRSS scheme and consider the MLEs of the location and scale parameters when sampling from a location-scale family of distributions. The MLEs using VLRSS are compared with the MLEs based on SRS and RSS schemes.

The rest of the paper is organized as follows. In Section 2, we consider the MLEs using VLRSS, and also derive the corresponding Fisher information matrices. A numerical study is conducted in Section 3 in order to gain insight into the performances of the proposed and existing MLEs. Finally, Section 4 concludes the paper.

\section{Maximum Likelihood estimation}

In this section, we consider the MLEs for the unknown parameters (location and scale) of a location-scale family of distributions under the VLRSS scheme.

Let $X_{1}, X_{2}, \ldots, X_{n}$ be a random sample of size $n$ from a location-scale family of distributions with probability density function (PDF) $(1 / \sigma) f((x-\mu) / \sigma)$ and cumulative distribution function (CDF) $F((x-\mu) / \sigma)$, where $\mu$ and $\sigma$ are the location and scale parameters, respectively. The likelihood function under SRS is

$$
L=\frac{1}{\sigma^{n}} \prod_{i=1}^{n} f\left(Z_{i}\right)
$$

where $Z_{i}=\left(X_{i}-\mu\right) / \sigma$.

From equation (2.1) the log-likelihood function is

$$
\ln (L)=-n \ln (\sigma)+\sum_{i=1}^{n} \ln f\left(Z_{i}\right)
$$

Let $X_{(1: n)}, X_{(2: n)}, \ldots, X_{(n: n)}$ be the ordered statistics obtained from a simple random sample of size $n$. Then the PDF and CDF of the $i$ th order statistic $X_{(i: n)}(1 \leq i \leq n)$ are respectively, given by

$$
\begin{aligned}
g\left(Z_{(i: n)}\right) & =\frac{n !}{\sigma(i-1) !(n-i) !}\left\{F\left(Z_{(i: n)}\right)\right\}^{i-1}\left\{1-F\left(Z_{(i: n)}\right)\right\}^{n-i} f\left(Z_{(i: n)}\right), \\
G\left(Z_{(i: n)}\right) & =\sum_{r=i}^{n}\left(\begin{array}{l}
n \\
r
\end{array}\right)\left\{F\left(Z_{(i: n)}\right)\right\}^{r}\left\{1-F\left(Z_{(i: n)}\right)\right\}^{n-r},
\end{aligned}
$$

where $Z_{(i: n)}=\left(X_{(i: n)}-\mu\right) / \sigma$.

The likelihood function under the VLRSS scheme is defined as

$$
L^{*}=\prod_{j=1}^{r}\left\{\prod_{i=1}^{k} g\left(Z_{i\left(v: m_{1}\right) j}\right) \prod_{i=k+1}^{2 k} g\left(Z_{i\left(m_{1}-v+1: m_{1}\right) j}\right) \prod_{i=k+1}^{m-k} g\left(Z_{i+k(i: m) j}\right)\right\}
$$

where $Z_{i\left(v^{*}: m^{*}\right) j}=\left(X_{i\left(v^{*}: m^{*}\right) j}-\mu\right) / \sigma$ for $v^{*}=v$ or $v^{*}=m_{1}-v+1$ and $m^{*}=m_{1}$ or $m^{*}=m$. Here, $X_{i\left(v^{*}: m^{*}\right) j}$ is the $v^{*}$ th order statistic from the $i$ th sample of size $m^{*}$ in the $j$ th cycle, i.e., $X_{i\left(v^{*}: m^{*}\right) j}=v^{*}$ th $\min \left\{X_{i 1 j}, X_{i 2 j}, \ldots, X_{i m^{*} j}\right\}$.

Using equation (2.5), the log-likelihood function is

$$
\ln \left(L^{*}\right)=\sum_{j=1}^{r}\left\{\sum_{i=1}^{k} \ln g\left(Z_{i\left(v: m_{1}\right) j}\right)+\sum_{i=k+1}^{2 k} \ln g\left(Z_{i\left(m_{1}-v+1: m_{1}\right) j}\right)+\sum_{i=k+1}^{m-k} \ln g\left(Z_{i+k(i: m) j}\right)\right\} .
$$


Note here that for every fixed $i$ and $j, Z_{i\left(v^{*}: m^{*}\right) j}$ and $Z_{\left(v^{*}: m^{*}\right)}$ are identically distributed, i.e., $Z_{i\left(v^{*}: m^{*}\right) j} \stackrel{d}{=}$ $Z_{\left(v^{*}: m^{*}\right)}$. Using equations (2.3) and (2.4), equation (2.6) can be simplified as

$$
\begin{aligned}
\ln \left(L^{*}\right)= & c-m r \ln (\sigma)+\sum_{j=1}^{r} \sum_{i=1}^{k}\left\{(v-1) \ln F\left(Z_{i\left(v: m_{1}\right) j}\right)+\left(m_{1}-v\right) \ln \left\{1-F\left(Z_{i\left(v: m_{1}\right) j}\right)\right\}\right. \\
& \left.+\ln f\left(Z_{i\left(v: m_{1}\right) j}\right)\right\}+\sum_{j=1}^{r} \sum_{i=k+1}^{2 k}\left\{\left(m_{1}-v\right) \ln F\left(Z_{i\left(m_{1}-v+1: m_{1}\right) j}\right)\right. \\
& \left.+(v-1) \ln \left\{1-F\left(Z_{i\left(m_{1}-v+1: m_{1}\right) j}\right)\right\}+\ln f\left(Z_{i\left(m_{1}-v+1: m_{1}\right) j}\right)\right\} \\
& +\sum_{j=1}^{r} \sum_{i=k+1}^{m-k}\left\{(i-1) \ln F\left(Z_{i(i: m) j}\right)+(m-i) \ln \left\{1-F\left(Z_{i(i: m) j}\right)\right\}+\ln f\left(Z_{i(i: m) j}\right)\right\}
\end{aligned}
$$

where $C$ is a constant-independent of $\mu$ and $\sigma$.

\subsection{One parameter families}

Suppose that the scale parameter $\sigma$ is known. Then the MLEs of $\mu$ under SRS and VLRSS schemes, denoted by $\hat{\mu}_{\mathrm{SRS}}$ and $\hat{\mu}_{\mathrm{VLRSS}}$, are the solutions of the following equations, respectively,

$$
\frac{\partial \ln (L)}{\partial \mu}=-\frac{1}{\sigma} \sum_{i=1}^{n} \frac{f^{\prime}\left(Z_{i}\right)}{f\left(Z_{i}\right)}=0
$$

and

$$
\begin{aligned}
\frac{\partial \ln \left(L^{*}\right)}{\partial \mu}= & \frac{1}{\sigma} \sum_{j=1}^{r} \sum_{i=1}^{k}\left\{-(v-1) A_{i\left(v: m_{1}\right) j}^{(1)}+\left(m_{1}-v\right) A_{i\left(v: m_{1}\right) j}^{(2)}-A_{i\left(v: m_{1}\right) j}^{(3)}\right\} \\
& +\frac{1}{\sigma} \sum_{j=1}^{r} \sum_{i=k+1}^{2 k}\left\{-\left(m_{1}-v\right) A_{i\left(m_{1}-v+1: m_{1}\right) j}^{(1)}+(v-1) A_{i\left(m_{1}-v+1: m_{1}\right) j}^{(2)}-A_{i\left(m_{1}-v+1: m_{1}\right) j}^{(3)}\right\} \\
& +\frac{1}{\sigma} \sum_{j=1}^{r} \sum_{i=k+1}^{m-k}\left\{-(i-1) A_{i+k(i: m) j}^{(1)}+(m-i) A_{i+k(i: m) j}^{(2)}-A_{i+k(i: m) j}^{(3)}\right\}=0,
\end{aligned}
$$

where

$$
\begin{aligned}
& A_{i\left(t: m^{*}\right) j}^{(1)}=\frac{f\left(Z_{i\left(t: m^{*}\right) j}\right)}{F\left(Z_{i\left(t: m^{*}\right) j}\right)}, \\
& A_{i\left(t: m^{*}\right) j}^{(2)}=\frac{f\left(Z_{i\left(t: m^{*}\right) j}\right)}{1-F\left(Z_{i\left(t: m^{*}\right) j}\right)}, \\
& A_{i\left(t: m^{*}\right) j}^{(3)}=\frac{f^{\prime}\left(Z_{i\left(t: m^{*}\right) j}\right)}{f\left(Z_{i\left(t: m^{*}\right) j}\right)} .
\end{aligned}
$$

For the probability distributions satisfying the condition

$$
\frac{\partial}{\partial \mu} \int_{-\infty}^{\infty} f\left(\frac{x-\mu}{\sigma}\right) \mathrm{d} x=\int_{-\infty}^{\infty} \frac{\partial}{\partial \mu} f\left(\frac{x-\mu}{\sigma}\right) \mathrm{d} x
$$

it is well-known that the Fisher information about $\mu$ contained in a simple random sample of size $n$ can be obtained as

$$
I_{\mathrm{SRS}}^{*}(\mu)=-E_{Z}\left(\frac{\partial^{2} \ln (L)}{\partial \mu^{2}}\right)
$$


where $E_{Z}(\cdot)$ is the mathematical expectation with respect to $Z$. Similarly, under the VLRSS scheme, we have

$$
I_{\mathrm{VLRSS}}^{*}(\mu)=-E_{Z}\left(\frac{\partial^{2} \ln \left(L^{*}\right)}{\partial \mu^{2}}\right) .
$$

Thus we again differentiate equations (2.8) and (2.9) to obtain

$$
I_{\mathrm{SRS}}^{*}(\mu)=\frac{n}{\sigma^{2}} E_{Z}\left(\frac{f^{\prime}\left(Z_{i}\right)}{f\left(Z_{i}\right)}\right)^{2}
$$

and

$$
\begin{aligned}
I_{\mathrm{VLRSS}}^{*}(\mu)= & \frac{k}{\sigma^{2}} E_{Z}\left[-r(v-1) A_{i\left(v: m_{1}\right) j}^{(4)}+\left(m_{1}-v\right) A_{i\left(v: m_{1}\right) j}^{(5)}-r A_{i\left(v: m_{1}\right) j}^{(6)}\right] \\
& +\frac{r k}{\sigma^{2}} E_{Z}\left[-\left(m_{1}-v\right) A_{i\left(v: m_{1}-v+1\right) j}^{(4)}+(v-1) A_{i\left(v: m_{1}-v+1\right) j}^{(5)}-A_{i\left(v: m_{1}-v+1\right) j}^{(6)}\right] \\
& +\frac{r}{\sigma^{2}} \sum_{i=k+1}^{m-k} E_{Z}\left[-(i-1) A_{i(i: m-i+1) j}^{(4)}+(m-i) A_{i(i: m-i+1) j}^{(5)}-A_{i(i: m-i+1) j}^{(6)}\right],
\end{aligned}
$$

where

$$
\begin{aligned}
& A_{i\left(t: m^{*}\right) j}^{(4)}=\frac{f^{\prime}\left(Z_{i\left(t: m^{*}\right) j}\right)}{F\left(Z_{i\left(t: m^{*}\right) j}\right)}-\left(A_{i\left(t: m^{*}\right) j}^{(1)}\right)^{2} \\
& A_{i\left(t: m^{*}\right) j}^{(5)}=\frac{f^{\prime}\left(Z_{i\left(t: m^{*}\right) j}\right)}{1-F\left(Z_{i\left(t: m^{*}\right) j}\right)}+\left(A_{i\left(t: m^{*}\right) j}^{(2)}\right)^{2} \\
& A_{i\left(t: m^{*}\right) j}^{(6)}=\frac{f^{\prime \prime}\left(Z_{i\left(t: m^{*}\right) j}\right)}{f\left(Z_{i\left(t: m^{*}\right) j}\right)}-\left(A_{i\left(t: m^{*}\right) j}^{(3)}\right)^{2} .
\end{aligned}
$$

The asymptotic relative precision (RP) of $\hat{\mu}_{\mathrm{VLRSS}}$ with respect to $\hat{\mu}_{\mathrm{SRS}}$ is given by

$$
\lim _{r \rightarrow \infty} \operatorname{RP}\left(\hat{\mu}_{\mathrm{VLRSS}}, \hat{\mu}_{\mathrm{SRS}}\right)=\frac{I_{\mathrm{VLRSS}}^{*}(\mu)}{I_{\mathrm{SRS}}^{*}(\mu)},
$$

which turns out to be independent of $r$ and $\sigma$.

Now suppose that $\mu$ is known, and we wish to estimate $\sigma$ by the maximum likelihood method. Then the MLEs of $\sigma$ under SRS and VLRSS schemes, denoted by $\hat{\sigma}_{\mathrm{SRS}}$ and $\hat{\sigma}_{\mathrm{VLRSS}}$, are the solutions of the following equations, respectively,

$$
\frac{\partial \ln (L)}{\partial \sigma}=-\frac{n}{\sigma}-\frac{1}{\sigma} \sum_{i=1}^{n}\left(\frac{Z_{i} f^{\prime}\left(Z_{i}\right)}{f\left(Z_{i}\right)}\right)=0
$$

and

$$
\begin{aligned}
\frac{\partial \ln \left(L^{*}\right)}{\partial \sigma}= & -\frac{m r}{\sigma}+\frac{1}{\sigma} \sum_{j=1}^{r} \sum_{i=1}^{k}\left\{-(v-1) B_{i\left(v: m_{1}\right) j}^{(1)}+\left(m_{1}-v\right) B_{i\left(v: m_{1}\right) j}^{(2)}-B_{i\left(v: m_{1}\right) j}^{(3)}\right\} \\
& +\frac{1}{\sigma} \sum_{j=1}^{r} \sum_{i=k+1}^{2 k}\left\{-\left(m_{1}-v\right) B_{i\left(v: m_{1}-v+1\right) j}^{(1)}+(v-1) B_{i\left(v: m_{1}-v+1\right) j}^{(2)}-B_{i\left(v: m_{1}-v+1\right) j}^{(3)}\right\} \\
& +\frac{1}{\sigma} \sum_{j=1}^{r} \sum_{i=k+1}^{m-k}\left\{-(i-1) B_{i+k(i: m) j}^{(1)}+(m-i) B_{i+k(i: m) j}^{(2)}-B_{i+k(i: m) j}^{(3)}\right\}=0,
\end{aligned}
$$


TABLE 1 . Asymptotic RP of $\hat{\mu}_{\mathrm{VLRSS}}$ with respect to $\hat{\mu}_{\mathrm{SRS}}$ for $N(\mu, 1)$.

\begin{tabular}{|c|c|c|c|c|c|c|c|c|c|c|}
\hline \multirow[b]{3}{*}{$v \backslash m_{1}$} & \multicolumn{10}{|c|}{$\begin{array}{c}m=2 \text { and } k=0 \\
1.4805\end{array}$} \\
\hline & \multicolumn{10}{|c|}{$m=3$ and $k=1$} \\
\hline & 1 & 2 & 3 & 4 & 5 & 6 & 7 & 8 & 9 & 10 \\
\hline 1 & 1.4097 & 1.7301 & 1.9611 & 2.1449 & 2.2987 & 2.4315 & 2.5487 & 2.6538 & 2.7491 & 2.8365 \\
\hline 2 & $\longrightarrow$ & 1.7301 & 2.2292 & 2.5968 & 2.8955 & 3.1499 & 3.3729 & 3.5722 & 3.7528 & 3.9181 \\
\hline 3 & & - & 1.9611 & 2.5968 & 3.0677 & 3.4532 & 3.7843 & 4.0768 & 4.3399 & 4.5798 \\
\hline 4 & - & - & - & 2.1449 & 2.8955 & 3.4532 & 3.9113 & 4.3062 & 4.6565 & 4.9728 \\
\hline \multirow[t]{2}{*}{5} & $\longrightarrow$ & $\longrightarrow$ & 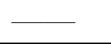 & - & 2.2987 & 3.1499 & 3.7843 & 4.3062 & 4.7570 & 5.1577 \\
\hline & \multicolumn{10}{|c|}{$m=4$ and $k=1$} \\
\hline$v \backslash m_{1}$ & 1 & 2 & 3 & 4 & 5 & 6 & 7 & 8 & 9 & 10 \\
\hline 1 & 1.8903 & 2.1306 & 2.3038 & 2.4416 & 2.5570 & 2.6566 & 2.7445 & 2.8233 & 2.8948 & 2.9603 \\
\hline 2 & & 2.1306 & 2.5049 & 2.7806 & 3.0046 & 3.1954 & 3.3627 & 3.5121 & 3.6475 & 3.7715 \\
\hline 3 & & & 2.3038 & 2.7806 & 3.1337 & 3.4229 & 3.6712 & 3.8906 & 4.0879 & 4.2678 \\
\hline 4 & & & - & 2.4416 & 3.0046 & 3.4229 & 3.7665 & 4.0627 & 4.3253 & 4.5626 \\
\hline \multirow[t]{2}{*}{5} & 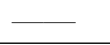 & 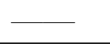 & 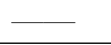 & - & 2.5570 & 3.1954 & 3.6712 & 4.0627 & 4.4008 & 4.7013 \\
\hline & \multicolumn{10}{|c|}{$m=5$ and $k=1$} \\
\hline$v \backslash m_{1}$ & 1 & 2 & 3 & 4 & 5 & 6 & 7 & 8 & 9 & 10 \\
\hline 1 & 2.3888 & 2.5810 & 2.7196 & 2.8299 & 2.9222 & 3.0018 & 3.0722 & 3.1352 & 3.1924 & 3.2449 \\
\hline 2 & & 2.5810 & 2.8805 & 3.1010 & 3.2802 & 3.4329 & 3.5667 & 3.6863 & 3.7946 & 3.8938 \\
\hline 3 & & - & 2.7196 & 3.1010 & 3.3836 & 3.6149 & 3.8136 & 3.9890 & 4.1469 & 4.2908 \\
\hline 4 & & & - & 2.8299 & 3.2802 & 3.6149 & 3.8897 & 4.1267 & 4.3368 & 4.5267 \\
\hline \multirow[t]{2}{*}{5} & $\square$ & $\square$ & 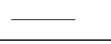 & $\longrightarrow$ & 2.9222 & 3.4329 & 3.8136 & 4.1267 & 4.3972 & 4.6376 \\
\hline & \multicolumn{10}{|c|}{$m=5$ and $k=2$} \\
\hline$v \backslash m_{1}$ & 1 & 2 & 3 & 4 & 5 & 6 & 7 & 8 & 9 & 10 \\
\hline 1 & 1.4974 & 1.8818 & 2.1590 & 2.3795 & 2.5641 & 2.7235 & 2.8641 & 2.9902 & 3.1046 & 3.2095 \\
\hline 2 & - & 1.8818 & 2.4808 & 2.9218 & 3.2802 & 3.5856 & 3.8532 & 4.0924 & 4.3090 & 4.5074 \\
\hline 3 & & $\longrightarrow$ & 2.1590 & 2.9218 & 3.4869 & 3.9496 & 4.3469 & 4.6978 & 5.0136 & 5.3014 \\
\hline 4 & $\longrightarrow$ & $\longrightarrow$ & $\longrightarrow$ & 2.3795 & 3.2802 & 3.9496 & 4.4993 & 4.9732 & 5.3934 & 5.7731 \\
\hline 5 & $\square$ & $\square$ & $\square$ & - & 2.5641 & 3.5856 & 4.3469 & 4.9732 & 5.5141 & 5.9950 \\
\hline
\end{tabular}

where

$$
\begin{aligned}
& B_{i\left(t: m^{*}\right) j}^{(1)}=Z_{i\left(t: m^{*}\right) j} A_{i\left(t: m^{*}\right) j}^{(1)}, \\
& B_{i\left(t: m^{*}\right) j}^{(2)}=Z_{i\left(t: m^{*}\right) j} A_{i\left(t: m^{*}\right) j}^{(2)}, \\
& B_{i\left(t: m^{*}\right) j}^{(3)}=Z_{i\left(t: m^{*}\right) j} A_{i\left(t: m^{*}\right) j}^{(3)} .
\end{aligned}
$$

The Fisher information about $\sigma$ contained in a simple random sample of size $n$ can be obtained as

$$
I_{\mathrm{SRS}}^{*}(\sigma)=-E_{Z}\left(\frac{\partial^{2} \ln (L)}{\partial \sigma^{2}}\right) .
$$

Similarly, under the VLRSS scheme, we have

$$
I_{\mathrm{VLRSS}}^{*}(\sigma)=-E_{Z}\left(\frac{\partial^{2} \ln \left(L^{*}\right)}{\partial \sigma^{2}}\right) .
$$

Thus we again differentiate equations (2.13) and (2.14) to obtain

$$
I_{\mathrm{SRS}}^{*}(\sigma)=\frac{n}{\sigma^{2}} E_{Z}\left\{\left(\frac{Z_{i} f^{\prime}\left(Z_{i}\right)}{f\left(Z_{i}\right)}\right)^{2}-1\right\}
$$


TABLE 2. Asymptotic RP of $\hat{\mu}_{\mathrm{VLRSS}}$ with respect to $\hat{\mu}_{\mathrm{SRS}}$ for $\operatorname{Logistic}(\mu, 1)$.

\begin{tabular}{|c|c|c|c|c|c|c|c|c|c|c|}
\hline & \multicolumn{10}{|c|}{$m=2$ and $k=0$} \\
\hline \multirow[b]{3}{*}{$v \backslash m_{1}$} & \multicolumn{10}{|c|}{1.5000} \\
\hline & \multicolumn{10}{|c|}{$m=3$ and $k=1$} \\
\hline & 1 & 2 & 3 & 4 & 5 & 6 & 7 & 8 & 9 & 10 \\
\hline 1 & 1.4667 & 1.8000 & 2.0000 & 2.1333 & 2.2286 & 2.3000 & 2.3556 & 2.4000 & 2.4364 & 2.4667 \\
\hline 2 & - & 1.8000 & 2.4000 & 2.8000 & 3.0857 & 3.3000 & 3.4667 & 3.6000 & 3.7091 & 3.8000 \\
\hline 3 & $\longrightarrow$ & - & 2.0000 & 2.8000 & 3.3714 & 3.8000 & 4.1333 & 4.4000 & 4.6182 & 4.8000 \\
\hline 4 & & - & - & 2.1333 & 3.0857 & 3.8000 & 4.3556 & 4.8000 & 5.1636 & 5.4667 \\
\hline \multirow[t]{2}{*}{5} & $\longrightarrow$ & $\longrightarrow$ & 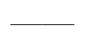 & $\longrightarrow$ & 2.2286 & 3.3000 & 4.1333 & 4.8000 & 5.3455 & 5.8000 \\
\hline & \multicolumn{10}{|c|}{$m=4$ and $k=1$} \\
\hline$v \backslash m_{1}$ & 1 & 2 & 3 & 4 & 5 & 6 & 7 & 8 & 9 & 10 \\
\hline 1 & 2.0000 & 2.2500 & 2.4000 & 2.5000 & 2.5714 & 2.6250 & 2.6667 & 2.7000 & 2.7273 & 2.7500 \\
\hline 2 & - & 2.2500 & 2.7000 & 3.0000 & 3.2143 & 3.3750 & 3.5000 & 3.6000 & 3.6818 & 3.7500 \\
\hline 3 & & & 2.4000 & 3.0000 & 3.4286 & 3.7500 & 4.0000 & 4.2000 & 4.3636 & 4.5000 \\
\hline 4 & & & - & 2.5000 & 3.2143 & 3.7500 & 4.1667 & 4.5000 & 4.7727 & 5.0000 \\
\hline \multirow[t]{2}{*}{5} & $\longrightarrow$ & $\longrightarrow$ & $\longrightarrow$ & - & 2.5714 & 3.3750 & 4.0000 & 4.5000 & 4.9091 & 5.2500 \\
\hline & \multicolumn{10}{|c|}{$m=5$ and $k=1$} \\
\hline$v \backslash m_{1}$ & 1 & 2 & 3 & 4 & 5 & 6 & 7 & 8 & 9 & 10 \\
\hline 1 & 2.5429 & 2.7429 & 2.8629 & 2.9429 & 3.0000 & 3.0429 & 3.0762 & 3.1029 & 3.1247 & 3.1429 \\
\hline 2 & - & 2.7429 & 3.1029 & 3.3429 & 3.5143 & 3.6429 & 3.7429 & 3.8229 & 3.8883 & 3.9429 \\
\hline 3 & & & 2.8629 & 3.3429 & 3.6857 & 3.9429 & 4.1429 & 4.3029 & 4.4338 & 4.5429 \\
\hline 4 & & & - & 2.9429 & 3.5143 & 3.9429 & 4.2762 & 4.5429 & 4.7610 & 4.9429 \\
\hline \multirow[t]{2}{*}{5} & & - & $\longrightarrow$ & $\longrightarrow$ & 3.0000 & 3.6429 & 4.1429 & 4.5429 & 4.8701 & 5.1429 \\
\hline & \multicolumn{10}{|c|}{$m=5$ and $k=2$} \\
\hline$v \backslash m_{1}$ & 1 & 2 & 3 & 4 & 5 & 6 & 7 & 8 & 9 & 10 \\
\hline 1 & 1.5714 & 1.9714 & 2.2114 & 2.3714 & 2.4857 & 2.5714 & 2.6381 & 2.6914 & 2.7351 & 2.7714 \\
\hline 2 & $\longrightarrow$ & 1.9714 & 2.6914 & 3.1714 & 3.5143 & 3.7714 & 3.9714 & 4.1314 & 4.2623 & 4.3714 \\
\hline 3 & & 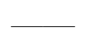 & 2.2114 & 3.1714 & 3.8571 & 4.3714 & 4.7714 & 5.0914 & 5.3532 & 5.5714 \\
\hline 4 & $\longrightarrow$ & $\longrightarrow$ & - & 2.3714 & 3.5143 & 4.3714 & 5.0381 & 5.5714 & 6.0078 & 6.3714 \\
\hline 5 & $\longrightarrow$ & $\longrightarrow$ & $\longrightarrow$ & $\longrightarrow$ & 2.4857 & 3.7714 & 4.7714 & 5.5714 & 6.2260 & 6.7714 \\
\hline
\end{tabular}

and

$$
\begin{aligned}
I_{\mathrm{VLRSS}}^{*}(\sigma)= & \frac{k}{\sigma^{2}} E_{Z}\left[-r(v-1) C_{i\left(v: m_{1}\right) j}^{(1)}+\left(m_{1}-v\right) C_{i\left(v: m_{1}\right) j}^{(2)}-C_{i\left(v: m_{1}\right) j}^{(3)}\right] \\
& +\frac{k}{\sigma^{2}} E_{Z}\left[-r\left(m_{1}-v\right) C_{i\left(v: m_{1}-v+1\right) j}^{(1)}+(v-1) C_{i\left(v: m_{1}-v+1\right) j}^{(2)}-r C_{i\left(v: m_{1}-v+1\right) j}^{(3)}\right] \\
& +\frac{1}{\sigma^{2}} \sum_{i=k+1}^{m-k} E_{Z}\left[-(i-1) C_{i+k(i: m) j}^{(1)}+(m-i) C_{i+k(i: m) j}^{(2)}-C_{i+k(i: m) j}^{(3)}\right],
\end{aligned}
$$

where

$$
\begin{aligned}
& C_{i\left(t: m^{*}\right) j}^{(1)}=Z_{i\left(t: m^{*}\right)}^{2} \frac{f^{\prime}\left(Z_{i\left(t: m^{*}\right) j}\right)}{F\left(Z_{i\left(t: m^{*}\right) j}\right)}+2 Z_{i\left(t: m^{*}\right)} A_{i\left(t: m^{*}\right) j}^{(1)}-\left(B_{i\left(t: m^{*}\right) j}^{(1)}\right)^{2}, \\
& C_{i\left(t: m^{*}\right) j}^{(2)}=Z_{i\left(t: m^{*}\right)}^{2} \frac{f^{\prime}\left(Z_{i\left(t: m^{*}\right) j}\right)}{1-F\left(Z_{i\left(t: m^{*}\right) j}\right)}+2 Z_{i\left(t: m^{*}\right)} A_{i\left(t: m^{*}\right) j}^{(2)}+\left(B_{i\left(t: m^{*}\right) j}^{(2)}\right)^{2}, \\
& C_{i\left(t: m^{*}\right) j}^{(3)}=Z_{i\left(t: m^{*}\right)}^{2} \frac{f^{\prime \prime}\left(Z_{i\left(t: m^{*}\right) j}\right)}{f\left(Z_{i\left(t: m^{*}\right) j}\right)}+2 Z_{i\left(t: m^{*}\right)} A_{i\left(t: m^{*}\right) j}^{(3)}-\left(B_{i\left(t: m^{*}\right) j}^{(3)}\right)^{2} .
\end{aligned}
$$


TABLE 3. Asymptotic RP of $\hat{\sigma}_{\mathrm{VLRSS}}$ with respect to $\hat{\sigma}_{\mathrm{SRS}}$ for $N\left(0, \sigma^{2}\right)$.

\begin{tabular}{|c|c|c|c|c|c|c|c|c|c|c|}
\hline \multirow[b]{4}{*}{$v \backslash m_{1}$} & \multicolumn{10}{|c|}{$m=2$ and $k=0$} \\
\hline & \multicolumn{10}{|c|}{1.1350} \\
\hline & \multicolumn{10}{|c|}{$m=3$ and $k=1$} \\
\hline & 1 & 2 & 3 & 4 & 5 & 6 & 7 & 8 & 9 & 10 \\
\hline 1 & 0.9944 & 1.0844 & 1.2701 & 1.4845 & 1.7074 & 1.9308 & 2.1513 & 2.3673 & 2.5782 & 2.7836 \\
\hline 2 & - & 1.0844 & 0.9832 & 1.0445 & 1.1868 & 1.3733 & 1.5850 & 1.8115 & 2.0465 & 2.2861 \\
\hline 3 & & - & 1.2701 & 1.0445 & 0.9841 & 1.0295 & 1.1418 & 1.2980 & 1.4839 & 1.6904 \\
\hline 4 & - & - & - & 1.4845 & 1.1868 & 1.0295 & 0.9856 & 1.0214 & 1.1137 & 1.2466 \\
\hline \multirow[t]{2}{*}{5} & 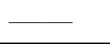 & $\longrightarrow$ & $\square$ & - & 1.7074 & 1.3733 & 1.1418 & 1.0214 & 0.9868 & 1.0164 \\
\hline & \multicolumn{10}{|c|}{$m=4$ and $k=1$} \\
\hline$v \backslash m_{1}$ & 1 & 2 & 3 & 4 & 5 & 6 & 7 & 8 & 9 & 10 \\
\hline 1 & 1.0376 & 1.1051 & 1.2443 & 1.4051 & 1.5723 & 1.7399 & 1.9052 & 2.0673 & 2.2254 & 2.3794 \\
\hline 2 & - & 1.1051 & 1.0292 & 1.0751 & 1.1819 & 1.3217 & 1.4805 & 1.6504 & 1.8266 & 2.0063 \\
\hline 3 & & & 1.2443 & 1.0751 & 1.0298 & 1.0638 & 1.1481 & 1.2652 & 1.4047 & 1.5595 \\
\hline 4 & & & - & 1.4051 & 1.1819 & 1.0638 & 1.0309 & 1.0578 & 1.1270 & 1.2267 \\
\hline \multirow[t]{2}{*}{5} & $\square$ & $\square$ & $\square$ & - & 1.5723 & 1.3217 & 1.1481 & 1.0578 & 1.0319 & 1.0541 \\
\hline & \multicolumn{10}{|c|}{$m=5$ and $k=1$} \\
\hline$v \backslash m_{1}$ & 1 & 2 & 3 & 4 & 5 & 6 & 7 & 8 & 9 & 10 \\
\hline 1 & 1.1124 & 1.1664 & 1.2778 & 1.4064 & 1.5401 & 1.6742 & 1.8065 & 1.9361 & 2.0626 & 2.1859 \\
\hline 2 & & 1.1664 & 1.1056 & 1.1424 & 1.2278 & 1.3397 & 1.4667 & 1.6026 & 1.7436 & 1.8874 \\
\hline 3 & & & 1.2778 & 1.1424 & 1.1062 & 1.1334 & 1.2008 & 1.2945 & 1.4061 & 1.5300 \\
\hline 4 & & & - & 1.4064 & 1.2278 & 1.1334 & 1.1071 & 1.1286 & 1.1839 & 1.2637 \\
\hline \multirow[t]{2}{*}{5} & $\longrightarrow$ & $\square$ & 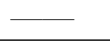 & $\square$ & 1.5401 & 1.3397 & 1.2008 & 1.1286 & 1.1078 & 1.1256 \\
\hline & \multicolumn{10}{|c|}{$m=5$ and $k=2$} \\
\hline$v \backslash m_{1}$ & 1 & 2 & 3 & 4 & 5 & 6 & 7 & 8 & 9 & 10 \\
\hline 1 & 0.9969 & 1.1049 & 1.3277 & 1.5850 & 1.8525 & 2.1206 & 2.3852 & 2.6444 & 2.8974 & 3.1439 \\
\hline 2 & - & 1.1049 & 0.9835 & 1.0570 & 1.2278 & 1.4516 & 1.7056 & 1.9774 & 2.2594 & 2.5469 \\
\hline 3 & & & 1.3277 & 1.0570 & 0.9845 & 1.0390 & 1.1737 & 1.3612 & 1.5843 & 1.8321 \\
\hline 4 & & & - & 1.5850 & 1.2278 & 1.0390 & 0.9863 & 1.0294 & 1.1400 & 1.2995 \\
\hline 5 & $\longrightarrow$ & $\square$ & $\longrightarrow$ & $\longrightarrow$ & 1.8525 & 1.4516 & 1.1737 & 1.0294 & 0.9878 & 1.0233 \\
\hline
\end{tabular}

The asymptotic RP of $\hat{\sigma}_{\mathrm{VLRSS}}$ with respect to $\hat{\sigma}_{\mathrm{SRS}}$ is given by

$$
\lim _{r \rightarrow \infty} \operatorname{RP}\left(\hat{\sigma}_{\mathrm{VLRSS}}, \hat{\sigma}_{\mathrm{SRS}}\right)=\frac{I_{\mathrm{VLRSS}}^{*}(\sigma)}{I_{\mathrm{SRS}}^{*}(\sigma)}
$$

which also turns out to be independent of $r$ and $\sigma$.

\subsection{Two-parameter families}

In order to compare the MLEs of $\mu$ and $\sigma$ under both SRS and VLRSS schemes simultaneously, the Fisher information matrices $\boldsymbol{I}_{\mathrm{SRS}}^{*}(\mu, \sigma)$ and $\boldsymbol{I}_{\mathrm{VLRSS}}^{*}(\mu, \sigma)$ are to be compared, given by

$$
\begin{aligned}
\boldsymbol{I}_{\mathrm{SRS}}^{*}(\mu, \sigma) & =\left[\begin{array}{cc}
I_{\mathrm{SRS}}^{*}(\mu) & I_{\mathrm{SRS}}^{*}(\mu, \sigma) \\
I_{\mathrm{SRS}}^{*}(\mu, \sigma) & I_{\mathrm{SRS}}^{*}(\sigma)
\end{array}\right] \\
\boldsymbol{I}_{\mathrm{VLRSS}}^{*}(\mu, \sigma) & =\left[\begin{array}{cc}
I_{\mathrm{VLRSS}}^{*}(\mu) & I_{\mathrm{VLRSS}}^{*}(\mu, \sigma) \\
I_{\mathrm{VLRSS}}^{*}(\mu, \sigma) & I_{\mathrm{VLRSS}}^{*}(\sigma)
\end{array}\right],
\end{aligned}
$$


TABLE 4. Asymptotic RP of $\hat{\sigma}_{\mathrm{VLRSS}}$ with respect to $\hat{\sigma}_{\mathrm{SRS}}$ for $\operatorname{Logistic}(0, \sigma)$.

\begin{tabular}{|c|c|c|c|c|c|c|c|c|c|c|}
\hline & \multicolumn{10}{|c|}{$m=2$ and $k=0$} \\
\hline & \multicolumn{10}{|c|}{1.1503} \\
\hline \multirow[b]{2}{*}{$v \backslash m_{1}$} & \multicolumn{10}{|c|}{$m=3$ and $k=1$} \\
\hline & 1 & 2 & 3 & 4 & 5 & 6 & 7 & 8 & 9 & 10 \\
\hline 1 & 1.0471 & 1.1473 & 1.3007 & 1.4806 & 1.6702 & 1.8610 & 2.0488 & 2.2316 & 2.4087 & 2.5798 \\
\hline 2 & - & 1.1473 & 1.1412 & 1.2149 & 1.3563 & 1.5400 & 1.7486 & 1.9708 & 2.1999 & 2.4315 \\
\hline 3 & $\longrightarrow$ & - & 1.3007 & 1.2149 & 1.1869 & 1.2436 & 1.3654 & 1.5328 & 1.7312 & 1.9504 \\
\hline 4 & 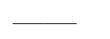 & - & - & 1.4806 & 1.3563 & 1.2436 & 1.2135 & 1.2594 & 1.3647 & 1.5146 \\
\hline \multirow[t]{2}{*}{5} & - & $\longrightarrow$ & $\longrightarrow$ & $\longrightarrow$ & 1.6702 & 1.5400 & 1.3654 & 1.2594 & 1.2309 & 1.2693 \\
\hline & \multicolumn{10}{|c|}{$m=4$ and $k=1$} \\
\hline$v \backslash m_{1}$ & 1 & 2 & 3 & 4 & 5 & 6 & 7 & 8 & 9 & 10 \\
\hline 1 & 1.1258 & 1.2010 & 1.3161 & 1.4510 & 1.5932 & 1.7363 & 1.8771 & 2.0143 & 2.1471 & 2.2754 \\
\hline 2 & - & 1.2010 & 1.1965 & 1.2517 & 1.3578 & 1.4956 & 1.6520 & 1.8187 & 1.9905 & 2.1641 \\
\hline 3 & & - & 1.3161 & 1.2517 & 1.2307 & 1.2732 & 1.3646 & 1.4901 & 1.6389 & 1.8033 \\
\hline 4 & & - & - & 1.4510 & 1.3578 & 1.2732 & 1.2507 & 1.2851 & 1.3641 & 1.4765 \\
\hline \multirow[t]{2}{*}{5} & - & $\longrightarrow$ & - & - & 1.5932 & 1.4956 & 1.3646 & 1.2851 & 1.2637 & 1.2925 \\
\hline & \multicolumn{10}{|c|}{$m=5$ and $k=1$} \\
\hline$v \backslash m_{1}$ & 1 & 2 & 3 & 4 & 5 & 6 & 7 & 8 & 9 & 10 \\
\hline 1 & 1.2275 & 1.2876 & 1.3796 & 1.4876 & 1.6014 & 1.7158 & 1.8285 & 1.9382 & 2.0445 & 2.1471 \\
\hline 2 & - & 1.2876 & 1.2840 & 1.3281 & 1.4130 & 1.5232 & 1.6484 & 1.7817 & 1.9192 & 2.0581 \\
\hline 3 & & & 1.3796 & 1.3281 & 1.3113 & 1.3454 & 1.4185 & 1.5189 & 1.6379 & 1.7694 \\
\hline 4 & - & - & - & 1.4876 & 1.4130 & 1.3454 & 1.3273 & 1.3548 & 1.4180 & 1.5080 \\
\hline \multirow[t]{2}{*}{5} & $\longrightarrow$ & $\longrightarrow$ & $\longrightarrow$ & - & 1.6014 & 1.5232 & 1.4185 & 1.3548 & 1.3377 & 1.3608 \\
\hline & \multicolumn{10}{|c|}{$m=5$ and $k=2$} \\
\hline$v \backslash m_{1}$ & 1 & 2 & 3 & 4 & 5 & 6 & 7 & 8 & 9 & 10 \\
\hline 1 & 1.0419 & 1.1622 & 1.3463 & 1.5622 & 1.7897 & 2.0186 & 2.2440 & 2.4634 & 2.6759 & 2.8812 \\
\hline 2 & - & 1.1622 & 1.1549 & 1.2433 & 1.4130 & 1.6335 & 1.8837 & 2.1505 & 2.4254 & 2.7032 \\
\hline 3 & - & - & 1.3463 & 1.2433 & 1.2097 & 1.2778 & 1.4239 & 1.6248 & 1.8629 & 2.1259 \\
\hline 4 & $\longrightarrow$ & $\longrightarrow$ & - & 1.5622 & 1.4130 & 1.2778 & 1.2417 & 1.2967 & 1.4231 & 1.6030 \\
\hline 5 & $\longrightarrow$ & $\longrightarrow$ & $\longrightarrow$ & - & 1.7897 & 1.6335 & 1.4239 & 1.2967 & 1.2625 & 1.3086 \\
\hline
\end{tabular}

where

$$
\begin{gathered}
I_{\mathrm{SRS}}^{*}(\mu, \sigma)=-E_{Z}\left(\frac{\partial^{2} \ln (L)}{\partial \sigma \partial \mu}\right), \\
I_{\mathrm{VLRSS}}^{*}(\mu, \sigma)=-E_{Z}\left(\frac{\partial^{2} \ln \left(L^{*}\right)}{\partial \sigma \partial \mu}\right) .
\end{gathered}
$$

After some simplifications, we obtain

$$
I_{\mathrm{SRS}}^{*}(\mu, \sigma)=\frac{n}{\sigma^{2}} E_{Z}\left\{Z_{i}\left(\frac{f^{\prime}\left(Z_{i}\right)}{f\left(Z_{i}\right)}\right)^{2}\right\}
$$

and

$$
\begin{aligned}
I_{\mathrm{VLRSS}}^{*}(\mu, \sigma)= & \frac{k}{\sigma^{2}} E_{Z}\left[-r(v-1) D_{i\left(v: m_{1}\right) j}^{(1)}+\left(m_{1}-v\right) D_{i\left(v: m_{1}\right) j}^{(2)}-r D_{i\left(v: m_{1}\right) j}^{(3)}\right] \\
& +\frac{r k}{\sigma^{2}} E_{Z}\left[-\left(m_{1}-v\right) D_{i\left(v: m_{1}-v+1\right) j}^{(1)}+(v-1) D_{i\left(v: m_{1}-v+1\right) j}^{(2)}-D_{i\left(v: m_{1}-v+1\right) j}^{(3)}\right] \\
& +\frac{r}{\sigma^{2}} \sum_{i=k+1}^{m-k} E_{Z}\left[-(i-1) D_{i+k(i: m) j}^{(1)}+(m-i) D_{i+k(i: m) j}^{(2)}-D_{i+k(i: m) j}^{(3)}\right]
\end{aligned}
$$


TABLE 5. Asymptotic RP of $\hat{\sigma}_{\mathrm{VLRSS}}$ with respect to $\hat{\sigma}_{\mathrm{VLRSS}}$ for $\operatorname{Exponential}(\sigma)$.

\begin{tabular}{|c|c|c|c|c|c|c|c|c|c|c|}
\hline \multirow[b]{4}{*}{$v \backslash m_{1}$} & \multicolumn{10}{|c|}{$m=2$ and $k=0$} \\
\hline & \multicolumn{10}{|c|}{1.4041} \\
\hline & \multicolumn{10}{|c|}{$m=3$ and $k=1$} \\
\hline & 1 & 2 & 3 & 4 & 5 & 6 & 7 & 8 & 9 & 10 \\
\hline 1 & 1.3082 & 1.5776 & 1.8082 & 2.0119 & 2.1956 & 2.3635 & 2.5187 & 2.6633 & 2.7990 & 2.9269 \\
\hline 2 & - & 1.5776 & 1.9247 & 2.2210 & 2.4900 & 2.7391 & 2.9722 & 3.1918 & 3.3999 & 3.5977 \\
\hline 3 & & - & 1.8082 & 2.2210 & 2.5582 & 2.8632 & 3.1477 & 3.4164 & 3.6721 & 3.9166 \\
\hline 4 & - & - & - & 2.0119 & 2.4900 & 2.8632 & 3.1956 & 3.5048 & 3.7975 & 4.0773 \\
\hline \multirow[t]{2}{*}{5} & 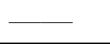 & $\longrightarrow$ & $\square$ & - & 2.1956 & 2.7391 & 3.1477 & 3.5048 & 3.8344 & 4.1460 \\
\hline & \multicolumn{10}{|c|}{$m=4$ and $k=1$} \\
\hline$v \backslash m_{1}$ & 1 & 2 & 3 & 4 & 5 & 6 & 7 & 8 & 9 & 10 \\
\hline 1 & 1.6846 & 1.8866 & 2.0596 & 2.2123 & 2.3501 & 2.4760 & 2.5924 & 2.7009 & 2.8026 & 2.8986 \\
\hline 2 & & 1.8866 & 2.1469 & 2.3691 & 2.5709 & 2.7577 & 2.9325 & 3.0973 & 3.2533 & 3.4017 \\
\hline 3 & & & 2.0596 & 2.3691 & 2.6221 & 2.8508 & 3.0641 & 3.2657 & 3.4575 & 3.6408 \\
\hline 4 & & - & - & 2.2123 & 2.5709 & 2.8508 & 3.1001 & 3.3320 & 3.5516 & 3.7614 \\
\hline \multirow[t]{2}{*}{5} & $\square$ & $\square$ & $\square$ & - & 2.3501 & 2.7577 & 3.0641 & 3.3320 & 3.5792 & 3.8129 \\
\hline & \multicolumn{10}{|c|}{$m=5$ and $k=1$} \\
\hline$v \backslash m_{1}$ & 1 & 2 & 3 & 4 & 5 & 6 & 7 & 8 & 9 & 10 \\
\hline 1 & 2.0840 & 2.2457 & 2.3840 & 2.5063 & 2.6165 & 2.7172 & 2.8103 & 2.8971 & 2.9785 & 3.0553 \\
\hline 2 & & 2.2457 & 2.4539 & 2.6317 & 2.7931 & 2.9426 & 3.0824 & 3.2142 & 3.3390 & 3.4578 \\
\hline 3 & & & 2.3840 & 2.6317 & 2.8340 & 3.0170 & 3.1877 & 3.3490 & 3.5024 & 3.6491 \\
\hline 4 & & & - & 2.5063 & 2.7931 & 3.0170 & 3.2165 & 3.4020 & 3.5776 & 3.7455 \\
\hline \multirow[t]{2}{*}{5} & $\longrightarrow$ & 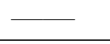 & 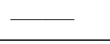 & $\longrightarrow$ & 2.6165 & 2.9426 & 3.1877 & 3.4020 & 3.5998 & 3.7867 \\
\hline & \multicolumn{10}{|c|}{$m=5$ and $k=2$} \\
\hline$v \backslash m_{1}$ & 1 & 2 & 3 & 4 & 5 & 6 & 7 & 8 & 9 & 10 \\
\hline 1 & 1.3750 & 1.6983 & 1.9750 & 2.2194 & 2.4398 & 2.6413 & 2.8276 & 3.0011 & 3.1639 & 3.3174 \\
\hline 2 & - & 1.6983 & 2.1147 & 2.4703 & 2.7931 & 3.0920 & 3.3718 & 3.6353 & 3.8850 & 4.1224 \\
\hline 3 & & & 1.9750 & 2.4703 & 2.8750 & 3.2410 & 3.5823 & 3.9048 & 4.2117 & 4.5050 \\
\hline 4 & & $\longrightarrow$ & - & 2.2194 & 2.7931 & 3.2410 & 3.6399 & 4.0109 & 4.3622 & 4.6979 \\
\hline 5 & $\longrightarrow$ & $\square$ & $\longrightarrow$ & $\longrightarrow$ & 2.4398 & 3.0920 & 3.5823 & 4.0109 & 4.4065 & 4.7803 \\
\hline
\end{tabular}

where

$$
\begin{aligned}
& D_{i\left(t: m^{*}\right) j}^{(1)}=A_{i\left(t: m^{*}\right) j}^{(1)}+Z_{i\left(t: m^{*}\right) j} A_{i\left(t: m^{*}\right) j}^{(4)} \\
& D_{i\left(t: m^{*}\right) j}^{(2)}=A_{i\left(t: m^{*}\right) j}^{(2)}+Z_{i\left(t: m^{*}\right) j} A_{i\left(t: m^{*}\right) j}^{(5)} \\
& D_{i\left(t: m^{*}\right) j}^{(3)}=A_{i\left(t: m^{*}\right) j}^{(3)}+Z_{i\left(t: m^{*}\right) j} A_{i\left(t: m^{*}\right) j}^{(6)}
\end{aligned}
$$

The RP of $\boldsymbol{I}_{\mathrm{SRS}}^{*}(\mu, \sigma)$ with respect to $\boldsymbol{I}_{\mathrm{VLRSS}}^{*}(\mu, \sigma)$ is given by

$$
\operatorname{RP}\left(\boldsymbol{I}_{\mathrm{VLRSS}}^{*}(\mu, \sigma), \boldsymbol{I}_{\mathrm{SRS}}^{*}(\mu, \sigma)\right)=\frac{\operatorname{Det}\left(\boldsymbol{I}_{\mathrm{SRS}}^{*}(\mu, \sigma)\right)}{\operatorname{Det}\left(\boldsymbol{I}_{\mathrm{VLRSS}}^{*}(\mu, \sigma)\right)},
$$

where $\operatorname{Det}(\cdot)$ denotes the determinant of $(\cdot)$.

\section{NUMERICAL COMPARISONS}

In this section, we compare the RPs of the MLEs obtained under SRS and VLRSS schemes. The results of the Section 2 are applied to the normal, logistic and exponential distributions. The numerical values of the 
TABLE 6 . Asymptotic RP of $\left(\hat{\mu}_{\mathrm{VLRSS}}, \hat{\sigma}_{\mathrm{VLRSS}}\right)^{\prime}$ with respect to $\left(\hat{\mu}_{\mathrm{SRS}}, \hat{\sigma}_{\mathrm{SRS}}\right)^{\prime}$ for $N\left(\mu, \sigma^{2}\right)$.

\begin{tabular}{|c|c|c|c|c|c|c|c|c|c|c|}
\hline \multirow[b]{4}{*}{$v \backslash m_{1}$} & \multicolumn{10}{|c|}{$m=2$ and $k=0$} \\
\hline & \multicolumn{10}{|c|}{1.6805} \\
\hline & \multicolumn{10}{|c|}{$m=3$ and $k=1$} \\
\hline & 1 & 2 & 3 & 4 & 5 & 6 & 7 & 8 & 9 & 10 \\
\hline 1 & 1.4019 & 1.8762 & 2.4907 & 3.1840 & 3.9247 & 4.6948 & 5.4831 & 6.2824 & 7.0877 & 7.8956 \\
\hline 2 & & 1.8762 & 2.1918 & 2.7123 & 3.4364 & 4.3257 & 5.3462 & 6.4711 & 7.6800 & 8.9570 \\
\hline 3 & - & & 2.4907 & 2.7123 & 3.0189 & 3.5550 & 4.3209 & 5.2916 & 6.4401 & 7.7416 \\
\hline 4 & - & - & 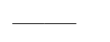 & 3.1840 & 3.4364 & 3.5550 & 3.8550 & 4.3986 & 5.1857 & 6.1992 \\
\hline \multirow[t]{2}{*}{5} & 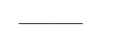 & - & - & 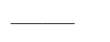 & 3.9247 & 4.3257 & 4.3209 & 4.3986 & 4.6945 & 5.2425 \\
\hline & \multicolumn{10}{|c|}{$m=4$ and $k=1$} \\
\hline$v \backslash m_{1}$ & 1 & 2 & 3 & 4 & 5 & 6 & 7 & 8 & 9 & 10 \\
\hline 1 & 1.9613 & 2.3544 & 2.8666 & 3.4307 & 4.0203 & 4.6221 & 5.2289 & 5.8365 & 6.4420 & 7.0439 \\
\hline 2 & & 2.3544 & 2.5780 & 2.9894 & 3.5510 & 4.2234 & 4.9785 & 5.7964 & 6.6627 & 7.5668 \\
\hline 3 & & & 2.8666 & 2.9894 & 3.2272 & 3.6414 & 4.2149 & 4.9225 & 5.7422 & 6.6558 \\
\hline 4 & - & - & - & 3.4307 & 3.5510 & 3.6414 & 3.8830 & 4.2976 & 4.8746 & 5.5970 \\
\hline \multirow[t]{2}{*}{5} & - & 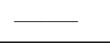 & 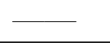 & 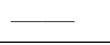 & 4.0203 & 4.2234 & 4.2149 & 4.2976 & 4.5411 & 4.9555 \\
\hline & \multicolumn{10}{|c|}{$m=5$ and $k=1$} \\
\hline$v \backslash m_{1}$ & 1 & 2 & 3 & 4 & 5 & 6 & 7 & 8 & 9 & 10 \\
\hline 1 & 2.6572 & 3.0104 & 3.4750 & 3.9799 & 4.5005 & 5.0257 & 5.5499 & 6.0702 & 6.5848 & 7.0928 \\
\hline 2 & & 3.0104 & 3.1848 & 3.5426 & 4.0275 & 4.5990 & 5.2314 & 5.9077 & 6.6163 & 7.3490 \\
\hline 3 & - & - & 3.4750 & 3.5426 & 3.7428 & 4.0971 & 4.5793 & 5.1639 & 5.8308 & 6.5648 \\
\hline 4 & - & - & - & 3.9799 & 4.0275 & 4.0971 & 4.3062 & 4.6573 & 5.1345 & 5.7203 \\
\hline \multirow[t]{2}{*}{5} & 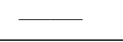 & 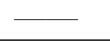 & 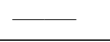 & - & 4.5005 & 4.5990 & 4.5793 & 4.6573 & 4.8713 & 5.2200 \\
\hline & \multicolumn{10}{|c|}{$m=5$ and $k=2$} \\
\hline$v \backslash m_{1}$ & 1 & 2 & 3 & 4 & 5 & 6 & 7 & 8 & 9 & 10 \\
\hline 1 & 1.4927 & 2.0793 & 2.8665 & 3.7715 & 4.7499 & 5.7754 & 6.8315 & 7.9074 & 8.9954 & 10.0903 \\
\hline 2 & & 2.0793 & 2.4398 & 3.0884 & 4.0275 & 5.2047 & 6.5722 & 8.0923 & 9.7358 & 11.4798 \\
\hline 3 & - & & 2.8665 & 3.0884 & 3.4329 & 4.1035 & 5.1021 & 6.3947 & 7.9431 & 9.7127 \\
\hline 4 & - & - & 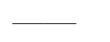 & 3.7715 & 4.0275 & 4.1035 & 4.4378 & 5.1192 & 6.1486 & 7.5024 \\
\hline 5 & - & - & - & 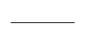 & 4.7499 & 5.2047 & 5.1021 & 5.1192 & 5.4471 & 6.1349 \\
\hline
\end{tabular}

asymptotic RPs of the MLEs based on VLRSS with respect to the MLEs based on SRS are computed using numerical integration in Mathematica 8.0, and are given in Tables 1-6. Various values of the sample sizes $m$, $m_{1}$ and $v$ are considered.

Based on Tables 1-6, the following remarks can be concluded:

- From Tables 1 and 2, for estimating the population mean of the normal and logistic distributions using VLRSS and SRS, it can be seen that the RP values are increasing as the values of $m_{1}$ and $v$ are increasing. As an example, for estimating the mean of the normal distribution, when $m_{1}=1$ and 10 , the RP values are 1.4097 and 2.8365, respectively when $v=1$. Also, when $m_{1}=9, v=1$ and 5 , the RP values are 2.7491 and 4.7570 , respectively.

- Tables 1 and 2, indicate that all values of the RPs are greater than one. This shows that the MLEs of the location parameter $\mu$ with VLRSS are more precise than those obtained with SRS. It is also observed that the MLEs under VLRSS turn out to be at least as efficient as the MLE with RSS when $m_{1} \geq m$.

- When estimating the standard deviation of the normal distribution, Table 3 revealed that the RP is increasing as $m_{1}$ values are increasing for fixed $v$, while it is decreasing as $v$ and $m$ values are increasing for fixed $m_{1}$ and $k$. On the other hand, when estimating the scale parameter $\sigma$ of normal and logistic distributions, from Tables 3 and 4 it is observed that, in most of the cases, the MLEs under VLRSS are more efficient that the 
MLEs with SRS. Similarly, for all sample sizes considered here, the MLEs with VLRSS also dominate the MLEs based on RSS when $m_{1} \geq m$ and $v=1$.

- Generally, we can say that the VLRSS is more efficient is estimating the location parameter than the scale parameter of the normal distribution for the same sample sizes. The same thing can be concluded for the case of the logistic distribution.

- For the case of one parameter as the scale parameter of the exponential distribution, a similar trend is observed in Table 5 as was seen in Tables 1 and 2. However, Table 5 shows that the RP values are increasing in $m_{1}$ for the same $v$ and $m$ and also it is increasing as $v$ values are increasing for fixed $m_{1}$. For illustration consider the case of $v=3$ with $m_{1}=3$ and 8 , the RP values are 2.0596 and 3.2567 , respectively when $m=4$. Also, the RP values are changing in $k$ for the same value of $m$. For example, when $m=5, v=2$, $m_{1}=4$, the RP values are 2.6317 when $k=1$ as compared to 2.4703 for $k=2$.

- In Table 6 the RPs are given when both parameters are unknown. It is worth mentioning here that the VLRSS-based MLEs outperform the MLEs based on the RSS scheme when $m_{1} \geq m$.

\section{Conclusion}

In this paper, we have obtained the MLEs of the location and scale parameters of a location-scale family of distributions under the VLRSS schemes. The MLEs with the VLRSS have been compared with the MLEs obtained under SRS and RSS schemes for some usual location-scale distributions. It turns out that with reasonable assumptions the MLEs under VLRSS are more precise than those obtained with SRS and RSS schemes. Thus we recommend the use of the proposed MLEs for efficient estimation of the location and scale parameters when sampling from a known location-scale family of distributions.

Acknowledgements. The author is grateful to the Editor and the anonymous referees for many of their valuable comments and suggestions which lead to this improved version of the paper.

\section{REFERENCES}

[1] A.D. Al-Naseer, L ranked set sampling: a generalized procedure for robust visual sampling. Commun. Stat.-Simul. Comput. 36 (2007) 33-43.

[2] A.D. Al-Nasser and A.I. Al-Omari, Minimax ranked set sampling. Rev. Invest. Oper. 39 (2018) 560-570.

[3] A.D. Al-Nasser, E. Ciavolino and A.I. Al-Omari, Extreme ranked repetitive sampling control charts. Pesquisa Oper. 40 (2020) $1-16$.

[4] A.I. Al-Omari, Estimation of mean based on modified robust extreme ranked set sampling. J. Stat. Comput. Simul. 81 (2011) $1055-1066$.

[5] A.I. Al-Omari and A.D. Al-Nasser, Ratio estimation using multistage median ranked set sampling approach. J. Stat. Theory Pract. 12 (2018) 512-529.

[6] A.I. Al-Omari and M.Z. Raqab, Estimation of the population mean and median using truncation-based ranked set samples. J. Stat. Comput. Simul. 83 (2013) 1453-1471.

[7] A.I. Al-Omari and E. Zamanzade, Goodness of fit tests for logistic distribution based on phi-divergence. Electron. J. Appl. Stat. Anal. 11 (2018) 185-195.

[8] A.I. Al-Omari, I.M. Almanjahie, A.S. Hassan and H.F. Nagy, Estimation of the stress-strength reliability for exponentiated pareto distribution using median and ranked set sampling methods. Comput. Mater. Continua 64 (2020) 835-857.

[9] S. Balci, A.D. Akkaya and B.E. Ulgen, Modified maximum likelihood estimators using ranked set sampling. J. Comput. Appl. Math. 238 (2013) 171-179.

[10] W. Chen, M. Xie and M. Wu, Parametric estimation for the scale parameter for scale distributions using moving extremes ranked set sampling. Stat. Probab. Lett. 83 (2013) 2060-2066.

[11] T.R. Dell and J.L. Clutter, Ranked set sampling theory with order statistics background. Biometrics 28 (1972) 545-555.

[12] A. Haq and A.I. Al-Omari, A new shewhart control chart for monitoring process mean based on partially ordered judgment subset sampling. Qual. Quantity 49 (2015) 1185-1202.

[13] A. Haq, J. Brown, E. Moltchanova and A.I. Al-Omari, Partial ranked set sampling design. Environmetrics 24 (2013) $201-207$.

[14] A. Haq, J. Brown, E. Moltchanova and A.I. Al-Omari, Mixed ranked set sampling design. J. Appl. Stat. 41 (2014) $2141-2156$.

[15] A. Haq, J. Brown, E. Moltchanova and A.I. Al-Omari, Varied L ranked set sampling scheme. J. Stat. Theory Pract. 9 (2015) $741-767$. 
[16] A. Haq, J. Brown, E. Moltchanova and A.I. Al-Omari, Paired double ranked set sampling. Commun. Stat.-Theory Methods 45 (2016) 2873-2889.

[17] A.A. Jemain, A.I. Al-Omari and K. Ibrahim, Multistage extreme ranked set samples for estimating the population mean. J. Stat. Theory App. 6 (2007) 456-471.

[18] A.A. Jemain, A.I. Al-Omari and K. Ibrahim, Multistage median ranked set sampling for estimating the population median. J. Math. Stat. 3 (2007) 58-64.

[19] G.A. McIntyre, A method for unbiased selective sampling, using ranked sets. Aust. J. Agr. Res. 3 (1952) 385-390.

[20] H.A. Muttlak, Median ranked set sampling. J. Appl. Stat. Sci. 6 (1997) 245-255.

[21] Y. Rui, H. Wangxue and Y. Dongsen, The efficiency of ranked set sampling design for parameter estimation for the log-extended exponential-geometric distribution. Iran. J. Sci. Technol. Trans. A: Sci. 44 (2020) 497-507.

[22] H.M. Samawi, M.S. Ahmed and W. Abu-Dayyeh, Estimating the population mean using extreme ranked set sampling. Biometrical J. 38 (1996) 577-586.

[23] M.H. Samuh, A.I. Al-Omari and N. Koyuncu, Estimation of the parameters of the new weibull-pareto distribution using ranked set sampling. Statistica 80 (2020) 103-123.

[24] L. Stokes, Parametric ranked set sampling. Ann. Inst. Stat. Math. 47 (1995) 465-482.

[25] K. Takahasi and K. Wakimoto, On unbiased estimates of the population mean based on the sample stratified by means of ordering. Ann. Inst. Stat. Math. 20 (1968) 1-31.

[26] E. Zamanzade and A.I. Al-Omari, New ranked set sampling for estimating the population man and variance. Hacettepe J. Math. Stat. 45 (2016) 1891-1905.

[27] E. Zamanzade and M. Mahdizadeh, Using ranked set sampling with extreme ranks in estimating the population proportion. Stat. Methods Med. Res. 29 (2020) 165-177.

[28] G. Zheng and M.F. Al-Saleh, Modified maximum likelihood estimators based on ranked set samples. Ann. Inst. Stat. Math. 54 (2002) 641-658. 